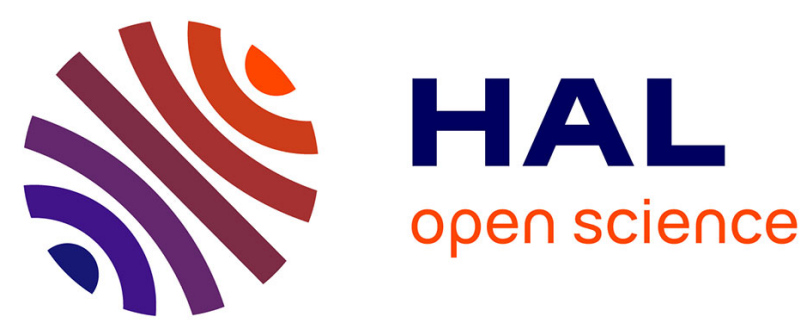

\title{
Place of bidirectional endoscopy in the assessment of iron deficiency in the elderly
}

Flora Ketz, Anne Buisson, Marine Levassort, Eric Pautas

\section{To cite this version:}

Flora Ketz, Anne Buisson, Marine Levassort, Eric Pautas. Place of bidirectional endoscopy in the assessment of iron deficiency in the elderly. Psychologie \& NeuroPsychiatrie du vieillissement, 2020, 18 (4), pp.377-383. 10.1684/pnv.2020.0891 . hal-03098132

\section{HAL Id: hal-03098132 \\ https://hal.sorbonne-universite.fr/hal-03098132}

Submitted on 5 Jan 2021

HAL is a multi-disciplinary open access archive for the deposit and dissemination of scientific research documents, whether they are published or not. The documents may come from teaching and research institutions in France or abroad, or from public or private research centers.
L'archive ouverte pluridisciplinaire HAL, est destinée au dépôt et à la diffusion de documents scientifiques de niveau recherche, publiés ou non, émanant des établissements d'enseignement et de recherche français ou étrangers, des laboratoires publics ou privés. 
Titre : Place des explorations digestives couplées dans le bilan d'une carence martiale chez le sujet âgé

Title : Place of bidirectional endoscopy in the assessment of iron deficiency in the elderly

Auteurs : Flora KETZ ${ }^{1,2}$, Anne BUISSON ${ }^{1,2}$, Marine LEVASSORT ${ }^{1}$, Eric PAUTAS $^{1,2}$

1 Service de Gériatrie Aiguë Polyvalente, hôpital Charles Foix, GH APHP Sorbonne Université ${ }^{2}$ UFR Médecine Sorbonne Université

\section{Correspondance}

Dr Flora KETZ

Service de Gériatrie Aiguë Polyvalente, hôpital Charles Foix, GH APHP Paris Sorbonne Université

7 avenue de la République, 94200 Ivry-sur-Seine

Tél : 0149594428

Fax : 0149594495

Courriel : flora.ketz@aphp.fr

Mots clés : carence martiale, endoscopies, sujet âgé

Keywords : iron deficiency, endoscopy, elderly

\section{Phrases clés :}

Les endoscopies digestives sont indiquées pour explorer les carences martiales des sujets âgés.

Il n’y pas de limite d’âge supérieur pour réaliser ces endoscopies. 
La rentabilité d'une exploration digestive couplée paraît démontrée, notamment chez les patients de plus de 80 ans.

Le taux de complication est faible, y compris chez les patients les plus âgés. 


\section{Résumé}

La carence martiale est une pathologie fréquente chez les patients âgés. Elle est le plus souvent due à des lésions digestives et se pose donc la question de la balance bénéficerisque et de la rentabilité des explorations digestives chez les patients âgés.

Les recommandations anglaises préconisent de réaliser des explorations digestives couplées (c'est-à-dire fibroscopie oeso-gastro-duodénale (FOGD) et coloscopie) dans le même temps, sans qu'il n'existe de limite d'âge supérieur. Dans les études faites chez les plus de 75 ans, la rentabilité diagnostique varie entre 63 et $68 \%$, ce qui est semblable à celle chez les plus jeunes, avec environ $40 \%$ de lésion haute et de lésion basse. La découverte simultanée de lésions digestives hautes et basses représente environ $10 \%$ des patients âgés. Les complications de la FOGD sont rares et essentiellement liées à l'anesthésie, tandis qu'il existe un petit surrisque de perforation chez les plus âgés, moins important en cas de coloscanner. Les contre-indications aux endoscopies digestives sont exceptionnelles. En cas d'endoscopies négatives, se posera la question de la poursuite ou non des explorations. 


\section{Summary :}

Iron deficiency is a common pathology in elderly patients. It is most often due to digestive lesions, so we wonder the benefit-risk balance and yield of digestive explorations.

The English recommendations recommend that bidirectional endoscopy

(esophagogastroduodenoscopy (EGD) and colonoscopy) be performed at the same time, without any upper age limit. In studies conducted in people over 75 years of age, the yield of diagnostic varies between 63 and $68 \%$, which is similar to that of younger people. There is about $40 \%$ of upper lesion and $40 \%$ of lower lesion. The simultaneous discovery of upper and lower digestive lesions represents about $10 \%$ of elderly patients. Complications of EGD are rare and mainly related to anesthesia. The main complication of colonoscopy is perforation and there is a small over-risk of perforation in older patients. This risk is less important when we use computed tomographic colonography. Contraindications of endoscopies are very rare. In case of negative endoscopies, we will wonder if we need further explorations or not. 


\section{Introduction}

L'anémie est une pathologie fréquemment rencontrée en pratique gériatrique. Sa prévalence est autour de 15 à $20 \%$ chez des sujets de plus de 75 ou 80 ans en population générale et peut atteindre $50 \%$ chez des patients du même âge hospitalisés ou institutionnalisés(1-3). La carence martiale est la première cause d'anémie en population générale représentant $50 \%$ des anémies dans le monde(4). Chez les patients âgés, la carence martiale participerait à environ un tiers des anémies, en sachant que les anémies multifactorielles sont plus fréquentes dans cette population(5-7).

En dehors des complications potentielles de l'anémie $(8,9)$, (la carence martiale entraine de nombreuses complications potentiellement invalidantes chez les sujets âgés, même quand elle n'a pas de retentissement hématologique : asthénie, syndrome dépressif, syndrome des jambes sans repos, troubles des phanères, insuffisance cardiaque et réhospitalisations précoces(1012). Il est donc important de penser à rechercher une carence martiale dans de nombreuses situations en Gériatrie. Si le gold-standard pour faire le diagnostic de carence martiale avec retentissement hématologique est le myélogramme, d'autres critères biologiques bien plus accessibles sont évidemment utilisés pour rechercher une carence martiale en pratique courante. Il est actuellement recommandé d'utiliser la ferritinémie et le coefficient de saturation de transferrine (CST)(13). Les seuils actuellement retenus pour définir une carence martiale sont : ferritinémie $<100 \mu \mathrm{g} / \mathrm{L}$ et/ou CST $<20 \%$ en l'absence de syndrome inflammatoire, et CST < $20 \%$ et ferritinémie entre 100 et $300 \mu \mathrm{g} / \mathrm{L}$ en cas de syndrome inflammatoire(14-17). La prévalence de la carence martiale est donc probablement largement sous-estimée dans la plupart des études qui utilisent fréquemment des seuils plus bas de ferritinémie $<15-50 \mu \mathrm{g} / \mathrm{L}$.

Une fois le diagnostic positif de carence martiale établi, se pose la question du bilan étiologique. On distingue maintenant deux types de carence martiale, qui ne sont pas 
exclusives entre elles et qui peuvent co-exister(18) : (i) les carences martiales fonctionnelles, principalement liées à un syndrome inflammatoire quel qu'en soit l'origine, situation dans laquelle le stock en fer de l'organisme n'est pas abaissé mais son utilisation est incorrecte principalement via l'augmentation de l'hepcidine(19) (ii) les carences martiales absolues par baisse du stock en fer, liées à une perte sanguine progressive, très majoritairement digestive, ou plus rarement liées à une malabsorption (infection à Helicobacter pylori, gastrite atrophique auto-immune, maladie cœliaque, maladie inflammatoire chronique, iatrogénie) voire à un manque d'apport(9,20).

En cas de perte sanguine digestive, l’origine est « haute » dans 40 à 60\% des cas (œsophagite, gastrite, ulcère, cancer, polype, angiodysplasie), «basse» dans 15 à $30 \%$ des cas (cancer colorectal, polype colique, angiodysplasie, lésion ano-rectale, maladie inflammatoire chronique), et non retrouvée dans 10 à 40\% des cas(9). La fréquence de ces lésions digestives est élevée chez les sujets âgés, pour qui se pose donc souvent la question de la place des explorations digestives en cas de découverte d'une carence martiale absolue : balance bénéfice-risque et rentabilité de ces explorations ? Cette brève revue de la littérature tente de donner quelques indications pratiques pour les gériatres confrontées à cette problématique.

\section{Recommandations concernant les explorations digestives en cas de carence martiale chez le sujet âgé}

L'exploration étiologique de la carence martiale n'a pas fait l'objet de recommandations françaises. Les recommandations anglaises préconisent, en cas de carence martiale absolue, de réaliser des endoscopies haute et basse couplées, c'est-à-dire fibroscopie œso-gastroduodénale (FOGD) et coloscopie dans le même temps, sauf s'il existe des arguments significatifs pour un saignement extra-digestif (recommandation de haut grade en cas d'anémie et recommandation de grade faible s'il n'y a pas d'anémie). Si la FODG est faite en 
première intention et retrouve une lésion potentiellement responsable, seule la présence d'un cancer gastrique doit faire surseoir à la coloscopie. Il n'y a par ailleurs pas de place pour la recherche de sang dans les selles en cas d'anémie par carence martiale(21).

Pour ce qui concerne la faisabilité des endoscopies digestives chez le sujet âgé, aucun âge limite n'a été proposé(22,23). Une évaluation par un gériatre peut s'avérer utile pour décider du caractère licite ou non des investigations digestives chez des patients comorbides, les experts recommandant une décision au cas par cas(22).

\section{Rentabilité diagnostique des explorations digestives couplées}

Le tableau I résume les résultats de différentes études évaluant la rentabilité des explorations digestives couplées en cas de carence martiale ; dans ces études les explorations digestives couplées s'entendent comme l'association de la FOGD à une coloscopie ou à un coloscanner, et la rentabilité diagnostique est définie par le pourcentage d'examens mettant en évidence une lésion potentiellement responsable de la carence. Dans les études dont les patients ont une moyenne d'âge de 75 ans ou plus, la rentabilité diagnostique varie entre 63 et $68 \%$, semblable à celle notée dans les travaux incluant des patients plus jeunes. La distribution relative des lésions hautes ou basses est équilibrée chez les sujets âgés, autour de $40 \%$ des cas pour chacune; les lésions basses sont proportionnellement plus souvent notées dans les cohortes gériatriques. La découverte simultanée de lésions digestives hautes et basses représente environ $10 \%$ des patients âgés ; dans une étude française menée chez des patients de plus de 80 ans ayant bénéficié d'endoscopies couplées pour carence martiale mais avec une anémie, on notait jusqu'à 43\% de lésions concomitantes aux deux étages(24).

Les études comparant directement deux populations de patients âgés et de patients plus jeunes, n'ont été faites que pour évaluer la rentabilité de la coloscopie seule (toutes 
indications confondues) (Tableau II). Leurs résultats plaident aussi en faveur de la plus grande rentabilité chez les sujets âgés.

La prévalence du cancer colorectal augmentant avec l'âge(25), il n'est pas surprenant d'observer que la rentabilité de la coloscopie pour ce diagnostic augmente avec l'âge (Tableaux I et II). Dans les cohortes de patients d'âge moyen ou médian supérieur à 80 ans, les explorations digestives couplées mènent ainsi à la découverte d'un cancer colo-rectal dans au moins un quart des cas.

Toutes ces études ne font qu'évaluer les explorations digestives couplées sur la rentabilité diagnostique définie comme la mise en évidence d'une lésion digestive, et posent la question de leur rentabilité en terme de survie voire de qualité de vie. Aucune étude ne s'est malheureusement intéressée à ces critères d'évaluation, qui seraient les plus utiles au clinicien au moment de prendre la décision de prescrire ces explorations digestives. Un élément de réponse, en cas de découverte d'une lésion néoplasique, est apporté par le travail de Hamaker et coll. qui décrit le devenir de 37 patients de plus de 80 ans dont le cancer colique a été diagnostiqué dans le bilan d'une anémie par carence martiale : un gain en survie après chirurgie du cancer colorectal est noté si l'espérance de vie au moment du diagnostic est d'au moins 1,3 ans(26).

\section{Faisabilité et complications des explorations digestives}

Dans les publications où elles sont évaluées pour des endoscopies digestives couplées, les complications rapportées sont rares, globalement retrouvées dans 0,2 à 2,8\% des cas(27-30). Seule une étude rétrospective chez 403 patients de plus de 80 ans trouve un taux de complications plus élevé avec 85 hypotensions ou bradycardies transitoires (soit $20 \%$ de la cohorte population)(31). 
Dans les études évaluant la FOGD seule, le taux de complications est très faible y compris chez les patients âgés. Elles sont aspécifiques, troubles du rythme cardiaque, bronchospasme, confusion, hypoxie transitoire...(27,32-34). Dans une étude spécifiquement gériatrique, Seinelä ne retrouve aucune complication majeure sur les 154 fibroscopies réalisées dans une cohorte de patients d'âge moyen 88,5 ans(35).

Pour ce qui est de la coloscopie, il n'y a pas d'élément dans la littérature permettant de retenir l'idée répandue que la préparation colique serait de moins bonne qualité chez les patients âges. Dans une méta-analyse de 20 études incluant des patients de plus de 65 ans passant une coloscopie toute indication confondue, un nombre similaire de coloscopies complètes est noté chez les plus de 65 ans et chez les plus de 80 ans (84\%), avec même d'avantage de mauvaises préparations chez les plus jeunes $(18,8 \%$ versus $12,1 \%)(36)$. En ce qui concerne les complications de la coloscopie, l'une des plus grandes craintes est la perforation perprocédure; cet évènement qui reste un des principaux freins aux explorations digestives couplées chez les patients âgés a cependant une incidence faible et le risque semble diminuer dans les travaux les plus récents(37-40). Dans une large étude rétrospective incluant plus de 100000 coloscopies réalisées chez des patients d'âge moyen de 69,4 ans, un risque de 33 perforations pour 100000 coloscopies est noté(41). Une autre étude de cohorte américaine menée chez les plus de 65 ans (moyenne d'âge 74 ans) trouve une incidence plus élevée de 196 perforations pour 100000 coloscopies. Enfin, une étude plus récente chez des sujets de plus de 65 ans retrouve 60 perforations pour 100000 coloscopies(42). Le risque de perforation augmente avec l'âge et le nombre de comorbidités ; ainsi, dans la méta-analyse déjà citée, évaluant les coloscopies toutes indications confondues, en comparant les 65-80 ans et les plus de 80 ans, les patients les plus âgés ont 1,7 fois plus de risque d'avoir une complication, toutes complications confondues (perforation, saignement, cardio-respiratoire), et notamment 1,6 fois plus de risque de faire une perforation(36). Le saignement per ou post-procédure, 
autre grande crainte liée à la coloscopie, apparaît lui aussi rare ; les études faites en population gériatrique montrent un risque de saignement entre $0,2 \%$ et $0,5 \%(28,30,31)$. En sus de ces chiffres bruts, plusieurs travaux se sont intéressés à comparer les complications de la coloscopie entre patients âgés et plus jeunes. Globalement, leurs résultats montrent un nombre d'effets indésirables mineurs plus important chez les patients âgés, mais pas de sur-risque de complications majeures(37,40,43-47). Dans une étude israélienne, aucune complication n'est même survenue chez 41 patients de plus de 90 ans (48). En comparant 110 patients de plus de 80 ans et 814 patients de moins de 80 ans bénéficiant d'une coloscopie, Arora et coll. notent un taux de complication comparable dans les 2 groupes, avec simplement une durée de procédure plus élevée pour les plus de 80 ans (22 vs 18,5 minute, $\mathrm{p}<0,0001)(49)$.

Le coloscanner est parfois, avec la FOGD, inclus dans une procédure d'explorations digestives couplées. Le risque de complications majeures, principalement perforation, est plus faible qu'avec une coloscopie mais ne doit pas être complètement occulté. Une méta-analyse incluant plus de 100000 patients, retrouve un taux de 40 perforations pour 100000 coloscanners(50). Une large étude rétrospective japonaise, incluant près de 150000 examens note un taux de 14 perforations pour 100000 coloscanners(51). Seules quelques études sur le coloscanner ont été conduites en population gériatrique. Une étude a notamment comparé les complications de 6114 coloscanners et 149202 coloscopies chez des patients de plus de 65 ans; le risque de saignement digestif et d'évènement cardiovasculaire apparait moins important après un coloscanner qu'une coloscopie, mais le taux de perforation était identique à 0,7 pour 1000 examens(52).

\section{Contre-indications aux endoscopies digestives}


Les contre-indications aux endoscopies digestives vont être soit liées à l'anesthésie, soit liées aux risques du geste endoscopique. Elles sont rarement absolues, et doivent être mis en balance avec le bénéfice attendu de l'examen.

Les contre-indications liées à l'anesthésie sont à la discrétion de l'anesthésiste qui évalue le patient, et sont liées à son état cardio-respiratoire et hémodynamique. Des explorations complémentaires peuvent être demandées avant l'anesthésie (échographie cardiaque, ...).

Les contre-indications liées aux gestes endoscopiques sont les contre-indications à l'insufflation colique : suspicion de perforation colique, de syndrome occlusif, de sigmoïdite diverticulaire, de colite aiguë, phase de cicatrisation post-polypectomie supra- centimétrique ou post-mucosectomie endoscopique. Il n'y a pas de contre-indication à la FOGD.

\section{Que faire en cas de négativité de FODG couplée à coloscopie ou coloscanner ?}

En cas de carence martiale, nous avons vu que les endoscopies digestives haute et basse identifient une cause potentielle pour $60 \%$ à $90 \%$ des patients. En cas d'endoscopies négatives, la question se pose de poursuivre ou non les explorations. Les recommandations de la British Society of Gastroenterology suggèrent qu'un examen complémentaire de l'intestin grêle n'est pas nécessaire d'emblée, mais doit être réalisé en l'absence de réponse à la supplémentation martiale (recommandations de grade B)(21). Il semble donc raisonnable de proposer dans un premier temps une supplémentation de la carence et une surveillance biologique.

En cas de carence martiale persistante ou récidivante, peut se discuter de répéter la gastroscopie, avec biopsie notamment à la recherche d'une cause de malabsorption. Si la coloscopie avait été incomplète ou de mauvaise qualité, peut aussi se discuter de la répéter. En effet jusqu'à $10 \%$ de lésions hautes et $7 \%$ de lésions basses peuvent avoir été manquées lors des premières explorations(53). Enfin, une exploration par vidéocapsule endoscopique 
(VCE) peut être proposée. Jusqu'à $80 \%$ des patients avec suspicion de saignement digestif chronique ont un diagnostic d'angiodysplasie grêlique après VCE(54). La VCE ne paraît pas avoir des résultats différents chez les patients âgés(55).

Dans une étude rétrospective française, menée chez des patients de plus de 65 ans avec carence martiale persistante, les endoscopies «second-look» et une VCE ont permis un diagnostic dans $63 \%$ des cas et un changement de traitement pour $58 \%$ des patients(56). A l'inverse, d'autres auteurs discutent la rentabilité de cette attitude en population gériatrique, du fait de fréquentes difficulté à préciser la localisation de la lésion et absence de retentissement thérapeutique(57).

\section{Conclusion}

Compte tenu de la grande fréquence de la carence martiale, avec ou sans anémie, dans la population gériatrique, se pose très souvent la question de son bilan étiologique dans lequel les explorations digestives (FODG et coloscopie ou coloscanner) sont en première ligne. La rentabilité d'une exploration digestive couplée paraît démontrée si on considère le nombre de lésions digestives mises en évidence avec cette attitude, notamment chez les patients de plus de 80 ans chez qui la fréquence d'un diagnostic de néoplasie digestive est élevée. Le taux faible de complications majeures liées à ces explorations digestives couplées, y compris pour les patients les plus âgés, incite à ne pas les récuser sur le simple critère de l'âge. Reste que cette rentabilité diagnostique mérite d'être mise en perspective avec une rentabilité en termes de survie et/ou de qualité de vie, sans que les données de la littérature ne permettent d'aider à l'évaluation de la balance bénéfice-risque intégrant ces notions. Dans les cas les plus complexes, du fait d'antécédents digestifs ou de pathologies cardio-pulmonaires pouvant majorer le risque, une concertation pluridisciplinaire peut être utile, une évaluation gériatrique y ayant toute sa place. 


\section{Bibliographie}

1. Eisenstaedt R, Penninx BWJH, Woodman RC. Anemia in the elderly: Current understanding and emerging concepts. Blood Rev 2006 ; 20:213-26.

2. Penninx BWJH, Pahor M, Cesari M, Corsi AM, Woodman RC, Bandinelli S, et al. Anemia is associated with disability and decreased physical performance and muscle strength in the elderly. J Am Geriatr Soc 2004 ; 52 : 719-24.

3. Deeruksa L, Sanchaisuriya K. Anemia in the elderly in Northeastern Thailand: a community-based study investigating prevalence, contributing factors, and hematologic features. Acta Haematol 2017 ; 138 : 96-102.

4. Vos T, Allen C, Arora M, Barber RM, Bhutta ZA, Brown A, et al. Global, regional, and national incidence, prevalence, and years lived with disability for 310 diseases and injuries, 1990-2015: a systematic analysis for the Global Burden of Disease Study 2015. Lancet $2016 ; 388: 1545-602$.

5. Pautas É, Siguret V, Kim TMA, Chaïbi P, Golmard J-L, Gouronnec A, et al. Anemia in the elderly: usefulness of an easy and comprehensive laboratory screen. Ann Biol Clin 2012 $; 70: 643-7$.

6. Petrosyan I, Blaison G, Andrès E, Federici L. Anaemia in the elderly: An aetiologic profile of a prospective cohort of 95 hospitalised patients. Eur J Intern Med 2012 ; 23 : $524-8$.

7. Çoban E, Timuragaoglu A, Meriç M. Iron deficiency anemia in the elderly: prevalence and endoscopic evaluation of the gastrointestinal tract in outpatients. Acta Haematol 2003 ; $110: 25-8$

8. Penninx BWJH, Pahor M, Woodman RC, Guralnik JM. Anemia in old age is associated with increased mortality and hospitalization. J Gerontol Med Sci 2006 ; 61 : 474-9. 
9. Andrès E, Serraj K, Federici L, Vogel T, Kaltenbach G. Anemia in elderly patients:

New insight into an old disorder. Geriatr Gerontol Int 2013 ; 13 : 519-27.

10. Stewart R, Hirani V. Relationship Between Depressive Symptoms, Anemia, and Iron Status in Older Residents From a National Survey Population. Psychosom Med $2012 ; 74$ : $208-13$.

11. Chassagne P, Bahri O, Roca F. Iron deficiency in elderly people. Clinical presentation and management. Ger Psychol Neuropsychiatr Vieil 2014 ; 12(S2) : 11-5.

12. Núñez J, Comín-Colet J, Miñana G, Núñez E, Santas E, Mollar A, et al. Iron deficiency and risk of early readmission following a hospitalization for acute heart failure. Eur J Heart Fail 2016; 18 : 798-802.

13. HAS. Diagnostic biologique d'une carence fer. 2011

14. Peyrin-Biroulet L, Williet N, Cacoub P. Guidelines on the diagnosis and treatment of iron deficiency across indications: a systematic review. Am J Clin Nutr 2015 ; 102 : 1585-94. 15. McMurray JJV, Adamopoulos S, Anker SD, Auricchio A, Bohm M, Dickstein K et al. ESC Guidelines for the diagnosis and treatment of acute and chronic heart failure 2012. Eur J Heart Fail $2012 ; 14: 803-69$.

16. Thomas DW, Hinchliffe RF, Briggs C, Macdougall IC, Littlewood T, Cavill I. Guideline for the laboratory diagnosis of functional iron deficiency. Br J Haematol $2013 ; 161$ :639-48.

17. Kis AM, Carnes M. Detecting iron deficiency in anemic patients with concomitant medical problems. J Gen Intern Med 1998 ; 13 : 455-61.

18. Le Petitcorps H, Monti A, Pautas É. Iron deficiency in elderly patients: use of biomarkers. Ann Biol Clin $2015 ; 73: 639-42$.

19. Camaschella C. Iron-Deficiency Anemia. N Engl J Med $2015 ; 372$ : 1832-43. 
20. Busti F, Campostrini N, Martinelli N, Girelli D. Iron deficiency in the elderly population, revisited in the hepcidin era. Front Pharmacol 2014 ; 5 (1-9).

21. Goddard AF, James MW, McIntyre AS, Scott BB, on behalf of the British Society of Gastroenterology. Guidelines for the management of iron deficiency anaemia. Gut $2011 ; 60$ : 1309-16.

22. Early DS, Acosta RD, Chandrasekhara V, Chathadi KV, Decker GA, Evans JA, et al. Modifications in endoscopic practice for the elderly. Gastrointest Endosc 2013 ; 78 : 1- 7. 23. de la Mora G, Marcon NE. Endoscopy in the elderly patient. Best Pract Res Clin Gastroenterol 2001; 15 : 999-1012.

24. Magnier G, Pautas E, Bornand-Rousselot A, Durand-Gasselin B, Saint-Jean O. Faisabilité, tolérance et intérêt de la gastroscopie et coloscopie couplées chez le sujet de plus de 80 ans. Rev Geriatr $1998 ; 23: 885-90$.

25. Purim O, Gordon N, Brenner B. Cancer of the colon and rectum: Potential effects of sex-age interactions on incidence and outcome. Med Sci Monit 2013 ; 19 : 203-9.

26. Hamaker ME, Acampo T, Remijn JA, van Tuyl SAC, Pronk A, van der Zaag ES, et al. Diagnostic choices and clinical outcomes in octogenarians and nonagenarians with irondeficiency anemia in the Netherlands. J Am Geriatr Soc 2013 ; 61 : 495-501.

27. Celik M. Efficacy of early endoscopy and colonoscopy in very elderly patients with gastrointestinal bleeding. Pak J Med Sci 2017 ; 33 : 187-90.

28. Duncan JE, Sweeney WB, Trudel JL, Madoff RD, Mellgren AF. Colonoscopy in the Elderly: Low Risk, Low Yield in Asymptomatic Patients. Dis Colon Rectum 2006 ; 49 : 646-51.

29. Fontagnier EM, Manegold BC. Die Koloskopie bei über 80-Jährigen. Dtsch Med Wschr $2000 ; 125: 1319-22$. 
30. Bat L, Pines A, Shemesh E, Levo Y, Zeeli D, Scapa E, et al. Colonoscopy in patients aged 80 years or older and its contribution to the evaluation of rectal bleeding. Postgrad Med $J 1992 ; 68: 355-8$.

31. Sardinha TC, Nogueras JJ, Ehrenpreis ED, Zeitman D, Estevez V, Weiss EG, et al. Colonoscopy in octogenarians: a review of 428 cases. Int J Colorectal Dis 1999 ; 14 : $172-6$.

32. Clarke GA, Jacobson BC, Hammett RJ, Carr-Locke DL. The indications, utilization and safety of gastrointestinal endoscopy in an extremely elderly patient cohort. Endoscopy $2001 ; 33: 580-4$.

33. Lockhart SP, Schofield PM, Gribble RJ, Baron JH. Upper gastrointestinal endoscopy in the elderly. Brit Med J $1985 ; 290: 283-283$.

34. Christe C, Janssens JP, Armenian B, Herrmann F, Vogt N. Midazolam sedation for upper gastrointestinal endoscopy in older persons: a randomized, double-blind, placebocontrolled study. J Am Geriatr Soc 2000 ; 48 : 1398-403.

35. Seinela L, Ahvenainen J, Ronneikko J, Haavisto M. Reasons for and outcome of upper gastrointestinal endoscopy in patients aged 85 years or more: retrospective study. Brit Med J $1998 ; 317: 575-575$.

36. Day LW, Kwon A, Inadomi JM, Walter LC, Somsouk M. Adverse events in older patients undergoing colonoscopy: a systematic review and meta-analysis. Gastrointest Endosc $2011 ; 74: 885-96$.

37. Lukens FJ, Loeb DS, Machicao VI, Achem SR, Picco MF. Colonoscopy in octogenarians: a prospective outpatient study. Am J Gastroenterol 2002 ; 97 : 1722-5.

38. Gatto NM, Frucht H, Sundararajan V, Jacobson JS, Grann VR, Neugut AI. Risk of perforation after colonoscopy and sigmoidoscopy: a population-based study. J Natl Cancer Inst $2003 ; 95: 230-6$. 
39. Arora G, Mannalithara A, Singh G, Gerson LB, Triadafilopoulos G. Risk of perforation from a colonoscopy in adults: a large population-based study. Gastrointest Endosc $2009 ; 69: 654-64$.

40. Miyanaga R, Hosoe N, Naganuma M, Hirata K, Fukuhara S, Nakazato Y, et al. Complications and outcomes of routine endoscopy in the very elderly. Endosc Int Open 2018 ; 06 : E224-9.

41. Avgerinos DV, Llaguna OH, Lo AY, Leitman IM. Evolving management of colonoscopic perforations. J Gastrointest Surg 2008 ; 12 : 1783-9.

42. Warren JL, Klabunde CN, Mariotto AB, Meekins A, Topor M, Brown ML, et al. Adverse events after outpatient colonoscopy in the Medicare population. Ann Intern Med $2009 ; 150: 849-58$.

43. Cabi MA, Achem SR, Kolts BE, McGee JB, MacMath T. Complications rates of colonoscopy in octagenarians: is age a risk factor? Cl Practice 1995 ; 108 : A7.

44. Ma W-T, Mahadeva S, Kunanayagam S, Poi PJ-H, Goh K-L. Colonoscopy in elderly Asians: A prospective evaluation in routine clinical practice. J Dig Dis 2007 ; 8 : 77-81.

45. Ure T, Dehghan K, Vernava AM, Longo WE, Andrus CA, Daniel GL. Colonoscopy in the elderly: Low risk, high yield. Surg Endosc 1995 ; 9 : 505-8.

46. Karajeh MA, Sanders DS, Hurlstone DP. Colonoscopy in elderly people is a safe procedure with a high diagnostic yield: a prospective comparative study of 2000 patients. Endoscopy $2006 ; 38: 226-30$.

47. Lagares-Garcia JA, Kurek S, Collier B, Diaz F, Schilli R, Richey J, et al. Colonoscopy in octogenarians and older patients. Surg Endosc $2001 ; 15$ : 262-5.

48. Schmilovitz-Weiss H, Weiss A, Boaz M, Levin I, Chervinski A, Shemesh E. Predictors of failed colonoscopy in nonagenarians: A single-center experience. J Clin Gastroenterol 2007 ; 41 : 388-93. 
49. Arora A, Singh P. Colonoscopy in patients 80 years of age and older is safe, with high success rate and diagnostic yield. Gastrointest Endosc 2004 ; 60 : 408-13.

50. Bellini D, Rengo M, De Cecco CN, Iafrate F, Hassan C, Laghi A. Perforation rate in CT colonography: a systematic review of the literature and meta-analysis. Eur Radiol 2014 ; $24: 1487-96$.

51. Nagata K, Takabayashi K, Yasuda T, Hirayama M, Endo S, Nozaki R, et al. Adverse events during CT colonography for screening, diagnosis and preoperative staging of colorectal cancer: a Japanese national survey. Eur Radiol 2017 ; 27 : 4970-8.

52. Zafar HM, Harhay MO, Yang J, Armstrong K. Adverse events following computed tomographic colonography compared to optical colonoscopy in the elderly. Prev Med Rep $2014 ; 1: 3-8$.

53. Polin V, Coriat R, Perkins G, Dhooge M, Abitbol V, Leblanc S, et al. Iron deficiency: From diagnosis to treatment. Dig Liver Dis 2013 ; 45 : 803-9.

54. Raju GS, Gerson L, Das A, Lewis B. American Gastroenterological Association (AGA) Institute medical position statement on obscure gastrointestinal bleeding. Gastroenterology 2007 ; 133 : $1694-6$.

55. Orlando G, Luppino IM, Lerose MA, Gervasi R, Amato B, Silecchia G, et al. Feasibility of capsule endoscopy in elderly patients with obscure gastrointestinal bleeding. An up-to-date report. BMC Surg 2012; 12 (Suppl 1) : S30.

56. Clere-Jehl R, Sauleau E, Ciuca S, Schaeffer M, Lopes A, Goichot B, et al. Outcome of endoscopy-negative iron deficiency anemia in patients above 65: A longitudinal multicenter cohort. Medicine 2016 ; 95 : 1-6.

57. Muhammad A, Vidyarthi G, Brady P. Role of small bowel capsule endoscopy in the diagnosis and management of iron deficiency anemia in elderly: A comprehensive review of the current literature. World J Gastroenterol $2014 ; 20: 8416-23$. 
58. Annibale B, Capurso G, Chistolini A, D’Ambra G, DiGiulio E, Monarca B, et al. Gastrointestinal causes of refractory iron deficiency anemia in patients without gastrointestinal symptoms. Am J Med $2001 ; 111: 439-45$.

59. Rockey DC, Cello JP. Evaluation of the gastrointestinal tract in patients with irondeficiency anemia. N Engl J Med 1993 ; 329 : 1691-5.

60. Kepczyk MT, Kadakia CSC. Prospective evaluation of gastrointestinal tract in patients with iron-deficiency anemia. Dig Dis Sci 1995 ; 40 : 1283-9.

61. Pongprasobchai S, Sripravoon T, Manatsathit S. Prospective evaluation of gastrointestinal lesions by bidirectional endoscopy in patients with iron deficiency anemia. $J$ Med Assoc Thai $2011 ; 94: 1321-6$.

62. Zuckerman G, Benitez J. A prospective study of bidirectional endoscopy (colonoscopy and upper endoscopy) in the evaluation of patients with occult gastrointestinal bleeding. Am J Gastroenterol $1992 ; 87: 62-6$.

63. Hardwick RH, Armstrong CP. Synchronous upper and lower gastrointestinal endoscopy is an effective method of investigating iron-deficiency anaemia. Br J Surg 1997 ; $84: 1725-8$.

64. Sari R, Aydogdu I, Sevinc A, Karincaoglu M. Upper and lower gastrointestinal endoscopical investigation in elderly patients with iron deficiency anaemia. Haematologica $2002 ; 31: 327-32$.

65. Niv E, Elis A, Zissin R, Naftali T, Novis B, Lishner M. Iron deficiency anemia in patients without gastrointestinal symptoms-a prospective study. Fam Pract 2005 ; 22 : 58-61.

66. Clere-Jehl R, Schaeffer M, Vogel T, Kiesmann M, Pasquali J-L, Andres E, et al. Upper and lower gastrointestinal endoscopies in patients over 85 years of age: Risk-benefit evaluation of a longitudinal cohort. Medicine 2017 ; $96: 1-7$.

67. Nahon S, Lahmek P, Aras N, Poupardin C, Lesgourgues B, Macaigne G, et al. 
Management and predictors of early mortality in elderly patients with iron deficiency anemia: a prospective study of 111 patients. Gastroenterol Clin Biol 2007 ; 31 : 169-74. 
Tableau I : Rentabilité des explorations digestives couplées dans le bilan étiologique d'une carence martiale

\begin{tabular}{|c|c|c|c|c|c|c|c|c|c|c|c|}
\hline Etude & $\begin{array}{c}\text { Annibale } \\
(\mathbf{5 8})\end{array}$ & $\begin{array}{l}\text { Rockey } \\
\quad(59)\end{array}$ & $\begin{array}{c}\text { Kepczyk } \\
\text { (60) }\end{array}$ & $\begin{array}{c}\text { Pongprasobchai } \\
(61)\end{array}$ & $\begin{array}{c}\text { Zuckerman } \\
\text { (62) }\end{array}$ & $\begin{array}{l}\text { Hardwick } \\
\quad(63)\end{array}$ & $\begin{array}{l}\text { Sari } \\
(64)\end{array}$ & $\begin{array}{c}\text { Coban } \\
(7)\end{array}$ & $\begin{array}{l}\text { Niv } \\
(65)\end{array}$ & $\begin{array}{l}\text { Clere- } \\
\text { Jehl } \\
(66)\end{array}$ & $\begin{array}{c}\text { Nahon } \\
(67)\end{array}$ \\
\hline Patients (n) & 71 & 100 & 70 & 103 & 100 & 89 & 95 & 96 & 48 & 55 & 111 \\
\hline $\begin{array}{c}\text { Age moyen } \\
(\mathbf{m} \pm \mathbf{D S})\end{array}$ & 59 & $60 \pm 14$ & 63,5 & $64 \pm 15$ & 65 & 65 & $65 \pm 12$ & $67 \pm 8$ & 75 & $88 \pm 2$ & $82 \pm 6$ \\
\hline Lésion haute & $59 \%$ & $36 \%$ & $56 \%$ & $43 \%$ & $36 \%$ & $57 \%$ & $58 \%$ & $57 \%$ & $35 \%$ & - & $40 \%$ \\
\hline Lésion basse & $25 \%$ & $25 \%$ & $30 \%$ & $25 \%$ & $26 \%$ & $58 \%$ & $11 \%$ & $27 \%$ & $37 \%$ & - & $39 \%$ \\
\hline $\begin{array}{l}\text { Lésions } \\
\text { couplées }\end{array}$ & $6 \%$ & $1 \%$ & $17 \%$ & $12 \%$ & $9 \%$ & $29 \%$ & 0 & $1 \%$ & $6 \%$ & - & $11 \%$ \\
\hline $\begin{array}{l}\text { Rentabilité } \\
\text { diagnostique }\end{array}$ & $85 \%$ & $62 \%$ & $71 \%$ & $56 \%$ & $53 \%$ & $84 \%$ & $68 \%$ & $84 \%$ & $63 \%$ & $64 \%$ & $68 \%$ \\
\hline $\begin{array}{c}\text { Cancer } \\
\text { colorectal }\end{array}$ & $14 \%$ & $11 \%$ & $6 \%$ & $13 \%$ & $6 \%$ & $35 \%$ & $5 \%$ & $8 \%$ & $10 \%$ & $27 \%^{\mathrm{a}}$ & $28 \%$ \\
\hline
\end{tabular}

$a$ : cancers haut et bas mélangés 
Tableau II : Etudes comparant la rentabilité de la coloscopie, toutes indications confondues, entre patients âgés et patients plus jeunes

\begin{tabular}{|c|c|c|c|}
\hline Etude & Karajeh (46) & Arora (49) & Ure (45) \\
\hline $1^{e r}$ groupe : $n$ (âge médian) & $\mathrm{n}=1000(75 \mathrm{ans})$ & $\mathrm{n}=110(83 \mathrm{ans})$ & $\mathrm{n}=354(77$ ans $)$ \\
\hline $2^{\text {nd }}$ groupe : $n$ (âge médian) & $\mathrm{n}=1000(54$ ans $)$ & $\mathrm{n}=814(60$ ans $)$ & $\mathrm{n}=302(60$ ans $)$ \\
\hline $\begin{array}{c}\text { Rentabilité globale } \\
1^{\text {er }} \text { groupe vs } 2^{\text {nd }} \text { groupe }\end{array}$ & $65 \%$ vs $45 \%(\mathrm{p}<0,0001)$ & $51 \%$ vs $37 \% *$ & $74 \%$ vs $60 \%(\mathrm{p}<0,05)$ \\
\hline $\begin{array}{l}\text { Diagnostic de cancer } \\
1^{\text {er }} \text { groupe vs } 2^{\text {nd }} \text { groupe }\end{array}$ & $\begin{array}{c}7,1 \% \text { vs } 1,3 \% \\
(p<0,0001)\end{array}$ & $\begin{array}{c}20 \% \text { vs } 7,4 \% \\
(p<0,0001)\end{array}$ & $6 \%$ vs $2 \%(\mathrm{p}<0,05)$ \\
\hline
\end{tabular}

* : significativité statistique non évaluée 